\title{
bFGF Activates Integrin $\beta 1$ and Subsequently Upregulates Collagens via the p-MEK1/2/p-ERK1/2 Signaling Pathway in L929 Cells
}

\section{Lian Yang}

Renmin Hospital of Wuhan University

\section{Shasha Hong}

Renmin Hospital of Wuhan University

Yang Li

Renmin Hospital of Wuhan University

\section{Tingting Wang}

Renmin Hospital of Wuhan University

\section{Li Hong ( $\nabla$ dr_hongli@whu.edu.cn )}

Renmin Hospital of Wuhan University

\section{Research Article}

Keywords: Pelvic organ prolapse, bFGF, integrin $\beta 1$, ERK1/2, Collagen

Posted Date: March 18th, 2021

DOI: https://doi.org/10.21203/rs.3.rs-296400/v1

License: (c) (1) This work is licensed under a Creative Commons Attribution 4.0 International License. Read Full License 
bFGF activates integrin $\beta 1$ and subsequently upregulates collagens via the $p$-MEK1/2/p-ERK1/2 signaling pathway in $L 929$ cells

Lian Yang $^{1 \triangleleft}$, Shasha Hong ${ }^{1 \triangleleft}$, Yang Li, Tingting Wang, Li Hong*

Affiliation: all authors come from the Department of Gynecology and Obstetrics, Renmin Hospital of Wuhan University, Wuhan,430060, Hubei Province, PR China

Lian Yang and Shasha Hong contributed equally.

* Address correspondence and reprint requests to Li Hong, Ph.D.

Department of Gynecology and Obstetrics, Renmin Hospital of Wuhan University, 238 Jiefang Road, Wuhan, Hubei Province 430060, P.R. China

Email: dr_hongli@whu.edu.cn; Tel: +86 027-88041911-82289 


\begin{abstract}
:
The substantial loss of collagen in the supporting tissues of the pelvic floor is characteristic of pelvic organ prolapse (POP). A bFGF-induced collagen increase has been widely recognized by scholars, but the role of bFGF in pelvic floor dysfunction and the mechanism by which bFGF promotes collagen have not been reported. Here, we elucidated this mechanism. After bFGF stimulation, L929 cells showed significantly increased expression of collagen, integrin $\beta 1$, and MEK1/2 signaling proteins. Our previous studies showed that integrin $\beta 1$ plays an important role in electric stimulation-induced collagen expression, which suggests an underlying mechanism. By overexpressing and silencing integrin $\beta 1$, we proved that integrin $\beta 1$ is also an important signal transduction protein of bFGF that promotes collagen through the MEK1/2 pathway, which is a classic collagen-promoting pathway. In summary, these findings suggest that bFGF can stimulate the expression of collagen through the integrin $\beta 1 / \mathrm{p}$-MEK1/2/p-ERK/1/2 signaling pathway in L929 cells.
\end{abstract}

\title{
Keyword
}

Pelvic organ prolapse; bFGF; integrin $\beta 1$; ERK1/2; Collagen 


\section{Introduction}

Female pelvic organ prolapse (POP), which is caused by a weakening of the supporting tissues of the pelvic floor, mainly involves uterine prolapse, vaginal anterior wall bulging, and vaginal posterior wall bulging[1]. POP affects adult women of all ages and causes major discomfort and a negative impact on daily life and work. Studies have shown that POP is closely associated with a reduction in collagen I and collagen III [2; 3; 4]. In recent years, promoting collagen regeneration has been an important strategy for pelvic floor treatment, such as electric stimulation[5; 6] [7].

Basic fibroblast growth factor (bFGF) was purified from bovine nervous tissue by Gospodarowicz in 1974[8]. It is secreted by various cells, such as fibroblasts, endothelial cells, and macrophages[9], and is widely used to repair wounds. In recent years, bFGF has been not only widely used in tissue engineering together with hydrogels and meshes, but also demonstrated potential in pelvic floor repair $[10 ; 11 ; 12]$ [13; 14]. bFGF not only promotes cell growth and differentiation but also strongly promotes collagen deposition[15; 16; 17]. Our previous studies have shown that mechanical forces can lead to apoptosis of fibroblasts and finally ECM changes[18]. Fibroblasts can spontaneously secrete bFGF, but no studies have examined changes in bFGF in the pelvic floor and the relationship between bFGF and POP. Therefore, we speculate that POP may cause changes in bFGF.

The mitogen-activated protein kinase (MAPK) pathway is a common pathway related to cell proliferation, inflammation, transformation, apoptosis, etc. MAPK signaling is also important in collagen production. MEK1/2 and ERK1/2 are important members of the MAPK pathway, and their phosphorylation reflects the activation of the MAPK pathway. Qiang et al.[19] reported that in liver fibrosis, the ERK signaling pathway plays an important role in the generation and degradation of ECM in the liver. Jaster, $\mathrm{R}$ et al. showed that ERK1/2 was involved in the development of fibrosis in multiple organs, such as the heart, liver, spleen, lung, and kidney[20;21; 22]. Integrin $\beta 1$, a cytoskeletal component belonging to the transmembrane receptor family, is an important signal transduction protein of cells that are widely distributed in smooth muscle cells, fibroblasts, and tumor cells. It is the main cell surface receptor that mediates extracellular matrix signaling. Our previous studies have shown that integrin $\beta 1$ is an important target for electrical stimulation-mediated induction of collagen production and plays a crucial role in the treatment of the pelvic floor by electrical stimulation[23], By chance, we also found that L929 cells cultured by bFGFshowed an unexpected increase in integrin $\beta 1$ expression, which had not been shown in previous studies, because both bFGF and integrin were thought to have their own ligands. In view of the importance of integrin $\beta 1$ in electrical-stimulation promoting collagen expression, and the effect of bFGF stimulating collagen expression, we urgently want to explore the mechanism of bFGF to promote collagen expression and whether integrin $\beta 1$ is involved in this process. 


\section{Materials and methods}

\section{Specimen collection}

POP group patients ( $\mathrm{n}=10$ ) were postmenopausal women whose POP-Q grade $\geq \mathrm{III}$ and underwent a vaginal hysterectomy in Renmin hospital of Wuhan university. The control group (NC-POP) selected postmenopausal women without cancer,connective tissue disease and those without a history of hormone replacement therapy $(\mathrm{n}=10)$. Ethical approval was taken from the Ethical Committee of the Renmin Hospital of Wuhan University. All patients signed the informed consent form. We choose the uterine sacral ligament (USL) to detect the protein expression.

\section{Cell culture and transduction}

L929 cells, a kind of mouse fibroblast cell, were purchased from China Center for Type Culture Collection (Wuhan). they were cultured in RPMI 1640 supplemented with $10 \%$ fetal bovine serum and $1 \%$ antibiotics at $37^{\circ} \mathrm{C}$ with $5 \% \mathrm{CO} 2$ in a humidified incubator. Mouse lentivirus vector (LV-Itgb1, Genechem, Shanghai, China) $(\mathrm{MOI}=60)$ and the infectious reagent HitransG P (40 ul) were added to set up the infection system. Similarly established the integrin $\beta 1$ silence system (Sh-Itgb1, MOI=50) and control system (Sh-Control, MOI=50), shRNA sequence of integrin $\beta 1$ : gcACGATGTGATGAT TTAGAA.

\section{Cell proliferation and apoptosis assay}

Cell Counting kit-8 (CCK8, biosharp, BS354B) was used to detect the proliferation of L929 cells at $0 \mathrm{ng} / \mathrm{ml}, 0.2 \mathrm{ng} / \mathrm{ml}, 2 \mathrm{ng} / \mathrm{ml}, 5 \mathrm{ng} / \mathrm{ml}$ and $10 \mathrm{ng} / \mathrm{ml}$ of bFGF at $0 \mathrm{~d}, 2 \mathrm{~d}, 4 \mathrm{~d}, 6 \mathrm{~d}$, and $8 \mathrm{~d}$. Cell apoptosis was detected by Apoptosis kit (Becton, Wuhan, USA).

\section{Western blots}

Extracted histone protein by liquid nitrogen milling, collected L929 cells by pancreatic enzyme digestion. Electrophoresis, electrotransfer, sealing, The primary antibody was incubated overnight (supplementary materials for information on primary resistance), incubated with HRP-labeled secondary antibodies for $1 \mathrm{~h}$. Chemiluminescence detection and imageJ analysis.

\section{Immunohistochemical}

Specimens were sealed with paraffin, then cut into $5 \mu \mathrm{m}$ slices and load. Dewaxing, baking, hydration, $3 \% \mathrm{H} 2 \mathrm{O} 2$ incubated, heated with PBS buffer, primary antibody overnight at $4{ }^{\circ} \mathrm{C}$, incubated by the secondary antibody (Maxim Biotechnologies, Fuzhou, China), stained by a DAB kit, used hematoxylin for 5 minutes and sealed piece after dehydration.

\section{Quantitative real-time polymerase chain reaction (qRT-PCR)}

Extracted RNA, cDNA was synthesized according to the instructions of the RevertAid First Strand cDNA Synthesis Kit(Thermo Fisher Scientific, Wuhan, China). Real-time PCR reaction was performed on the Applied Biosystems 7300/7500 real-time quantitative PCR instrument (Applied Biosystems, Thermo Fisher Scientific. China.). GAPDH was used as an internal control. The primer sequence is shown in Table I:

\section{Immunofluorescence}

$4 \%$ formaldehyde fixed L929 cells for 15 minutes and $0.5 \%$ Triton $\mathrm{x}-100$ permeated for 5 minutes, Closed by $5 \%$ goat serum for 30 minutes, and then incubated the primary antibody overnight at 
$4^{\circ} \mathrm{C}$. incubated with FITC-conjugated goat anti-rabbit IgG (1:200, Servicebio, Wuhan, China) the next day. The nucleus was stained by DAPI (Servicebio, Wuhan, China) for 5 min and then sealed piece.

\section{Statistical analysis}

All data were expressed as mean \pm SD. Usd SPSS 16.0 software (PSS Inc, Chicago, Illinois, USA) for statistical analysis. We choose the Kolmogorov-Smirnov test to evaluate the normality of the distribution and the t-test of unpaired students to compare the two groups. while the one-way ANOVA and Turkey test was used to compare the two groups. $\mathrm{P}<0.05$ was considered statistically significant. 


\section{Result}

The expression of bFGF, collagen III, collagen I, integrin $\beta 1$, p-MEK1/2, and p-ERK1/2 in patients with POP was lower than that in no-POP patients

Compared with that in the non-POP patients, the protein expression of bFGF, as well as the mRNA expression, was significantly decreased in the anterior vaginal walls of the patients with POP (Fig $1 \mathrm{a}, \mathrm{b}, \mathrm{d}, \mathrm{e})$. Immunohistochemical analysis showed that bFGF in the sacral ligament (brown staining) was more highly expressed in the non-POP patients than in the patients with POP (a). Besides, the expression of collagen III and collagen I decreased in the POP group ( $a, b)$, which is consistent with the findings in our previous studies[23]. Furthermore, the expression of integrin $\beta 1$, p-MEK1/2, and p-ERK1/2 showed a decrease in the POP group (b, c, d, e). These results indicate that there is a change in bFGF expression during the occurrence and development of POP, and POP not only affects the expression of collagen and integrin $\beta 1$ but also affects the phosphorylation of MEK1/2 and ERK1/2.
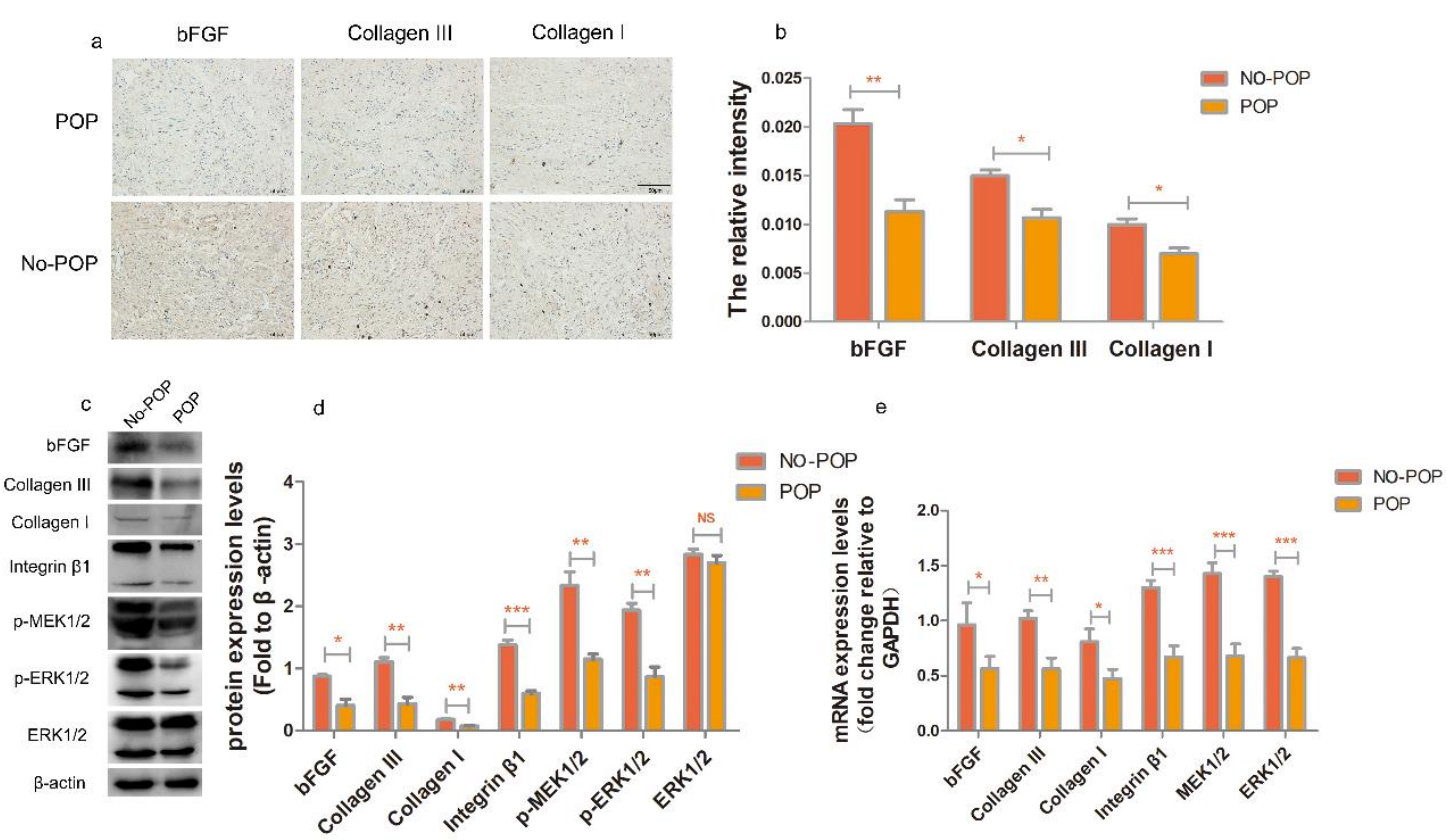

Fig 1: The expression of bFGF, Collagen III, Collagen I, Integrin $\beta 1$, p-MEK1/2, and p-ERK1/2 in POP patients was lower than NO-POP patients( POP group: POP patients; NO-POP: NO-POP patients. a: Immunohistochemical staining of the sacral ligament, amplification factor: $\times 200$. b: Fluorescence expression analysis of bFGF, Collagen III, and Collagen I. c: Protein expression of bFGF, CollagenIII, Collagen I, Integrin $\beta 1, \mathrm{p}-\mathrm{MEK} 1 / 2$ and $\mathrm{p}$-ERK1/2 in vaginal wall tissues. d: Statistical analysis of western blots. e: mRNA expression of bFGF, CollagenIII, Collagen I, Integrin $\beta 1$, p-MEK1/2, p-ERK1/2, and ERK1/2 in vaginal wall tissues. * represents $\mathrm{p}<0.05, * *$ represents $\mathrm{p}<0.01,{ }^{* * *}$ represents $\mathrm{p}<0.001$, NS represents no significance, Each experiment was repeated three times. 
bFGF promoted the expression of collagen and integrin $\beta 1$ and enhanced the phosphorylation of MEK1/2 and ERK1/2

Notably, we observed that an effective bFGF concentration can promote L929 cell proliferation, while a high dose inhibited this effect. To explore the optimal concentration of bFGF, we assessed 5 concentrations, $0 \mathrm{ng} / \mathrm{ml}, 0.2 \mathrm{ng} / \mathrm{ml}, 2 \mathrm{ng} / \mathrm{ml}, 5 \mathrm{ng} / \mathrm{ml}$ and $10 \mathrm{ng} / \mathrm{ml}$, in $96-$ well plates for the cell proliferation experiment. The cell proliferation rate was the highest when the concentration was 2 $\mathrm{ng} / \mathrm{ml}$ and the culture time was 2-4 $\mathrm{d}$, and a significant inhibitory effect was observed if the concentration was above $4 \mathrm{ng} / \mathrm{ml}$. (Fig $2 \mathrm{c}$ ). To investigate the effect of bFGF on apoptosis in L929 cells, we performed flow cytometry. We observed with the naked eye that the cells grew well and densely at low concentrations, while cell growth slowed and decreased at high concentrations. The apoptosis rate was significantly increased at high concentrations ( $8 \mathrm{ng} / \mathrm{ml}$ and $10 \mathrm{ng} / \mathrm{ml})$ (Fig $2 \mathrm{a}, \mathrm{b})$, which may be related to the cytotoxicity caused by high concentrations of the drug. To verify the relationship between bFGF and collagen, we stimulated L929 cells with $2 \mathrm{ng} / \mathrm{ml} \mathrm{bFGF}$ for $4 \mathrm{~d}$. Western blotting, cell immunofluorescence, and qRT-PCR were performed. The results showed that the collagen expression in the bFGF group was stronger than that in the control group. Western blotting, qRT-PCR, and immunofluorescence all showed the same results (Fig $2 \mathrm{~d}, \mathrm{e}, \mathrm{f}, \mathrm{g}$, $\mathrm{h}$ ), indicating that bFGF can effectively promote collagen expression. Furthermore, we found that the mRNA expression of MEK1/2 and ERK1/2 in the MAPK pathway, which is closely related to collagen production, increased after bFGF stimulation (Fig g). Western blot and immunofluorescence showed that bFGF enhanced the phosphorylation of MEK1/2 and ERK1/2(Fig $2 \mathrm{~d}$, e, f, h). As a major component of the cytoskeleton, integrin $\beta 1$ participates in signal transduction in many cells. To investigate whether bFGF affects integrin $\beta 1$, we detected the expression of integrin $\beta 1$. Notably, integrin $\beta 1$ in the bFGF stimulation group showed a significant increase (Fig $2 \mathrm{~d}$, e,).

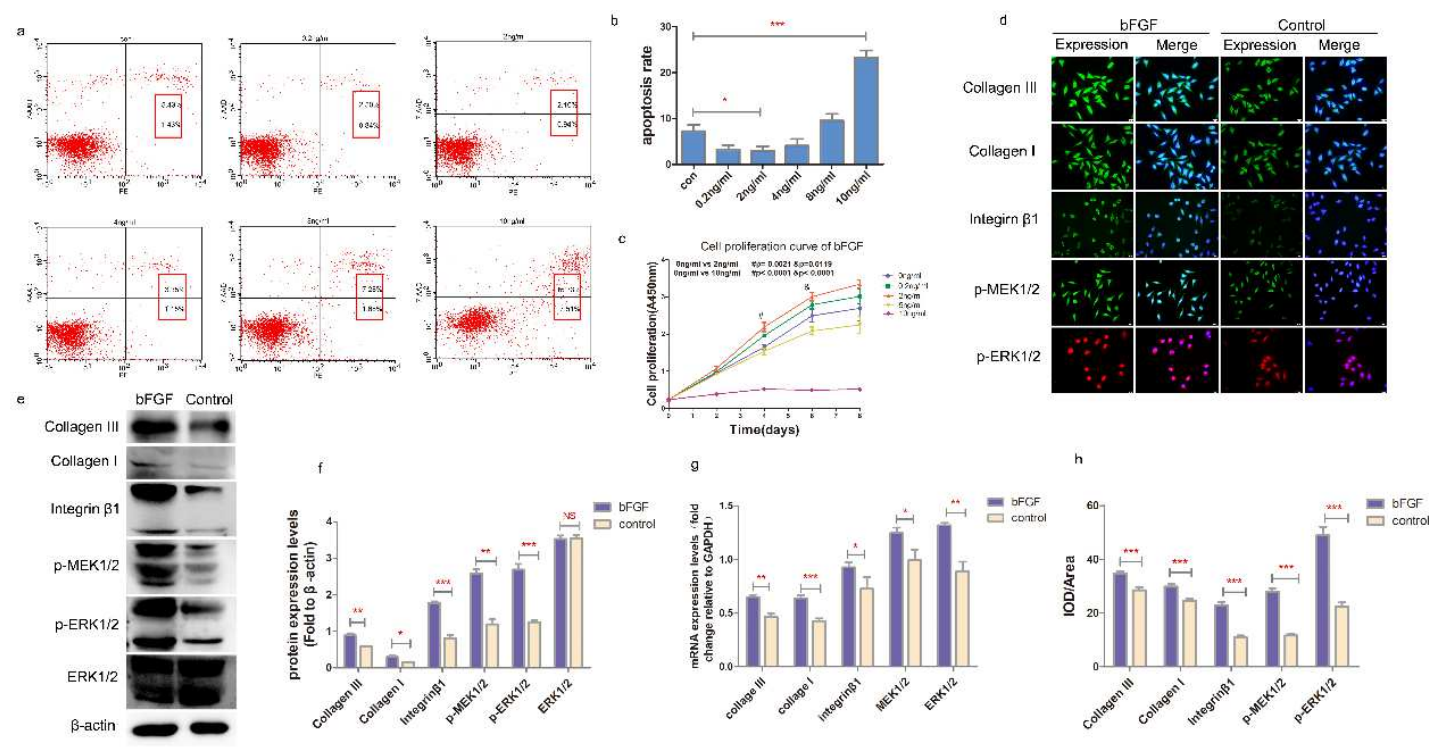

Fig 2: bFGF stimulation promoted the expression of Collagen and Integrin $\beta 1$ and enhance the phosphorylation of MEK1/2 and ERK1/2 a: Flow diagram of apoptosis; b: Statistical analysis apoptosis rate of $0 \mathrm{ng} / \mathrm{ml}, 0.2 \mathrm{ng} / \mathrm{ml}, 2 \mathrm{ng} / \mathrm{ml}, 4 \mathrm{ng} / \mathrm{ml}, 8 \mathrm{ng} / \mathrm{ml}$ and $10 \mathrm{ng} / \mathrm{ml}$, c: cell proliferation curve.d, h: Immunofluorescence staining and expression of cells stimulated by bFGF, amplification factor: $\times 400$; e,f: Protein expression of CollagenIII, collagen I, Integrin $\beta 1, \mathrm{p}-\mathrm{MEK} 1 / 2$, p-ERK1/2, and ERK1/2 after bFGF stimulation; g: mRNA expression of CollagenIII, collagen I, 
integrin $\beta 1, \mathrm{p}-\mathrm{MEK} 1 / 2, \mathrm{p}-\mathrm{ERK} 1 / 2$ and ERK1/2 after bFGF stimulation. $*$ represents $\mathrm{p}<0.05, * *$ represents $\mathrm{p}<0.01, * * *$ represents $\mathrm{p}<0.001$, NS represents no significance.

\section{bFGF regulated the phosphorylation of MEK1/2 and ERK1/2 and collagen expression through integrin $\beta 1$}

Integrin $\beta 1$ is a cytoskeletal protein that is important in signal transduction and participates in the interaction of intracellular cytoskeleton proteins. we hypothesized that bFGF stimulates integrin $\beta 1$ upstream of MEK1/2/ERK1/2 and regulates the phosphorylation of MEK1/2/ERK1/2. To test our hypothesis, we used lentivirus in L929 cells to establish an integrin $\beta 1$ overexpression and silencing system and then stimulated the cells with bFGF. The results confirmed that bFGF promoted the expression of integrin $\beta 1$ the phosphorylation of MEK1/2 and ERK1/2(Fig 3 a,c,d). The protein expression of integrin $\beta 1$ and collagen increased, similar to the immunofluorescence results (Fig $3 \mathrm{c}, \mathrm{d}, \mathrm{e}, \mathrm{f}$ ), while the silencing of integrin $\beta 1$ inhibited this effect (Fig $3 \mathrm{a}, \mathrm{c}, \mathrm{d}$ ). In addition, the overexpression of integrin $\beta 1$ mediated the increase in p-MEK1/2,p-ERK1/2,collagen I, and collagen III, which was supported by western blotting and immunofluorescence. This effect was further enhanced when bFGF was combined with the overexpression of integrin $\beta 1$. Therefore, bFGF can regulate the expression of $\mathrm{p}-\mathrm{MEK} 1 / 2$, p-ERK1/2, and collagen through integrin $\beta 1$.
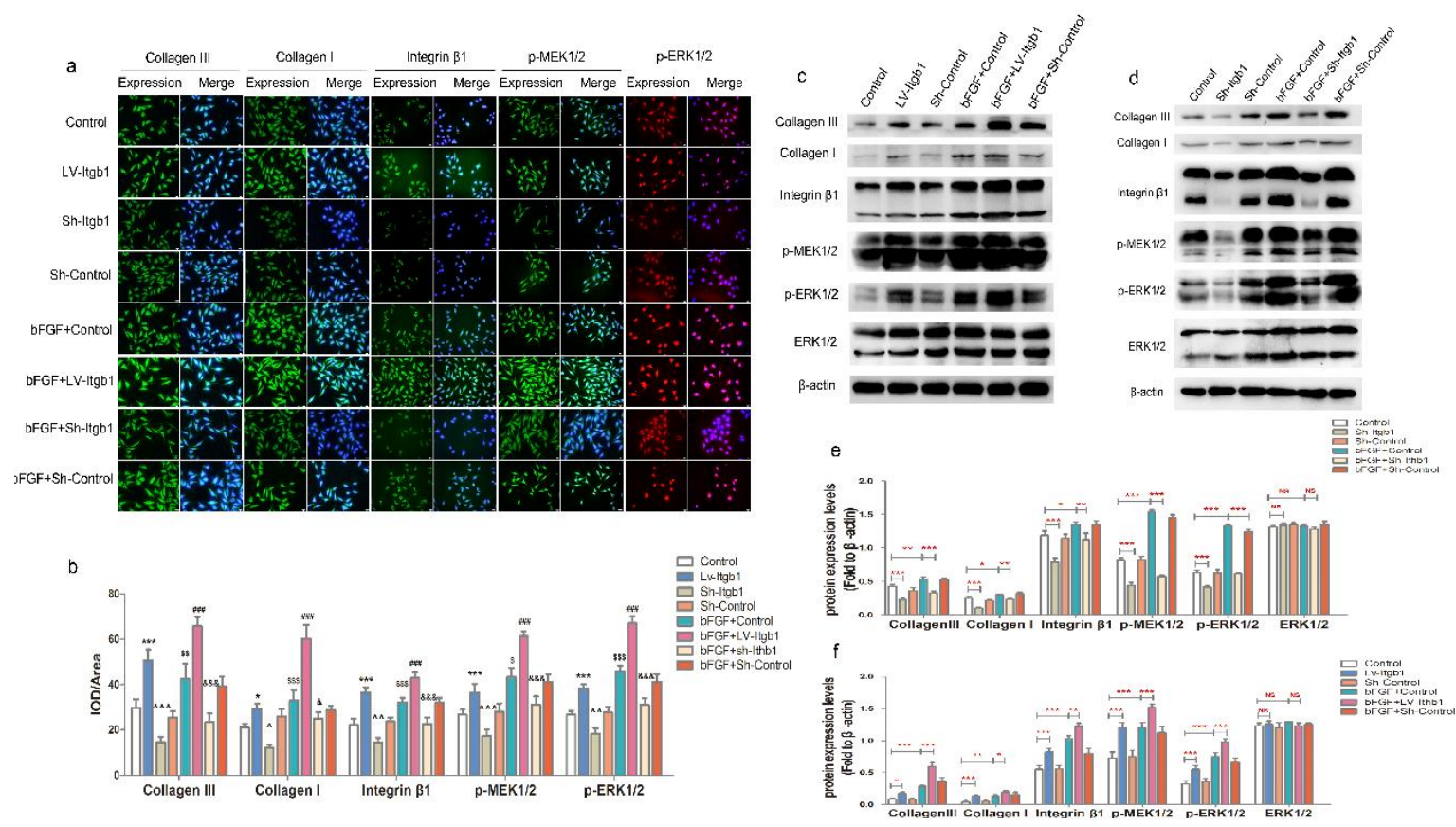

Fig 3: Fluorescence expression of CollagenIII, Collagen I, Integrin $\beta 1$, p-MEK1/2, P-ERK1/2 in cells after overexpression and silencing of integrin $\beta 1$. Control group: L929 cells cultivated by Conventional medium. LV-Itgb1: Integrins overexpress lentivirus transfected L929 cells. Sh-Itgb1: Integrins silence lentivirus transfected L929 cells. Sh-Control group: Lentivirus negative control group.bFGF+Control group: L929 cells cultivated by $2 \mathrm{ng} / \mathrm{ml}$ bFGF. bFGF+ LV-Itgb1 group: Integrins overexpress lentivirus transfected L929 cells and cultivated by $2 \mathrm{ng} / \mathrm{ml} \mathrm{bFGF}$. bFGF+Sh-Itgb1 group: Integrins silence lentivirus transfected L929 cells and cultivated by $2 \mathrm{ng} / \mathrm{ml}$ bFGF. bFGF+Sh-Control group: Lentivirus negative control group cultivated by $2 \mathrm{ng} / \mathrm{ml} \mathrm{bFGF}$. a: 
Fluorescence staining diagram, magnification factor: $\times 400$. b: Fluorescence expression analysis. *represents for Control VS Lv-Itgb1, ^ represents for control VS Sh-Itgb1, \$ represents for Control VS bFGF+control, \# represents bFGF+Control VS bFGF+ Lv-Itgb1, \& represents bFGF+Control VS bFGF+ Sh-Itgb1. c,f : Protein expression after integrin $\beta 1$ overexpression. d,e: Protein expression after integrin $\beta 1$ silence. A marker represents $p<0.05$, Two marks indicate $p$ $<0.01$, Three markers indicate $\mathrm{p}<0.001$, NS represents no significance.

\section{bFGF promoted collagen production through the MEK1/2/ERK1/2 signaling pathway}

To clarify that bFGF stimulates collagen production through the MEK1/2/ERK1/2 signal transduction pathway, we used the MEK1/2 inhibitor U0126 (CST, no. \#9903, China) $(10 \mu \mathrm{M})$ to treat L929 cells and then stimulated them with bFGF. We observed that the expression of collagen III and collagen I decreased significantly after the inhibition of MEK1/2. The fluorescence results were consistent with the western blot results (fig $4 \mathrm{a}, \mathrm{b}, \mathrm{c}, \mathrm{d}$ ).
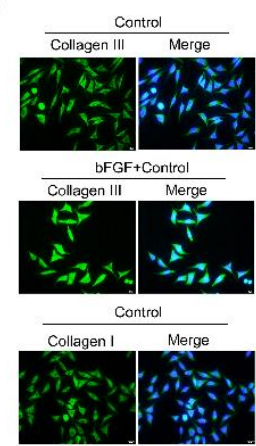

bFGF+Control
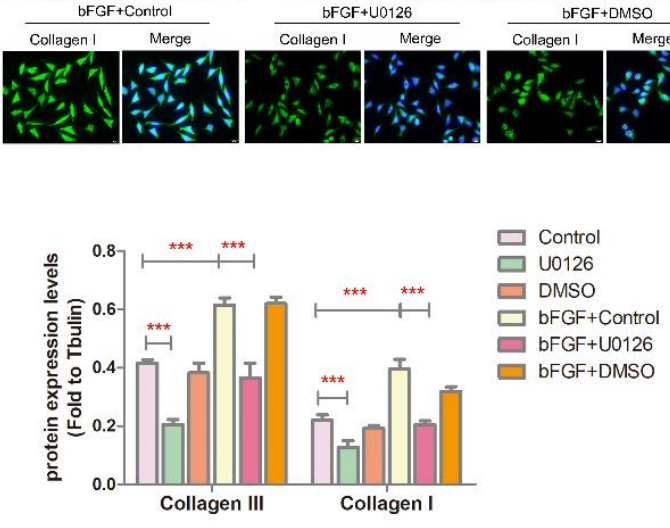

DMSO

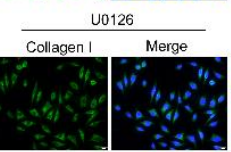

bFGF+U0126

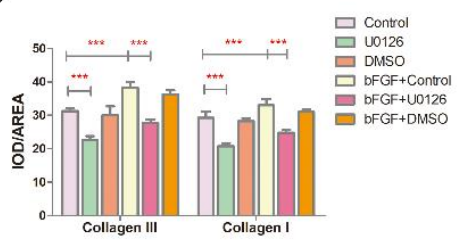

$\frac{\text { bFGF+U0126 }}{\text { Collagen III Merge }} \quad \frac{\text { bFGF+DMSO }}{\text { Collagen III Merge }}$
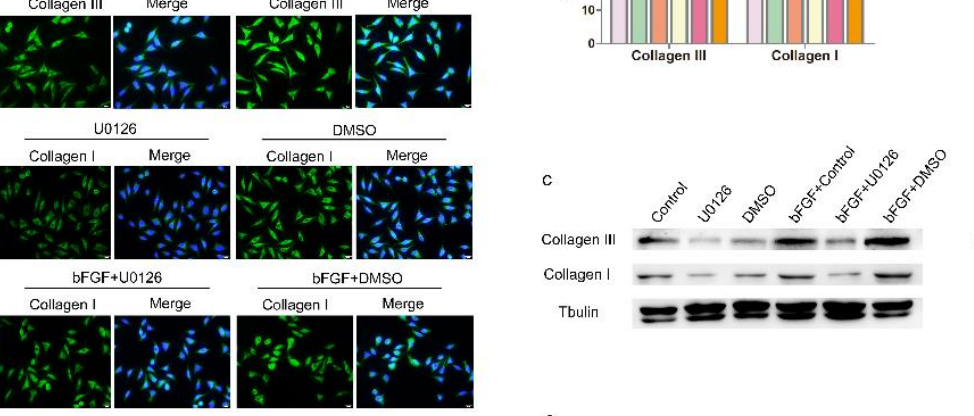

Tbulin

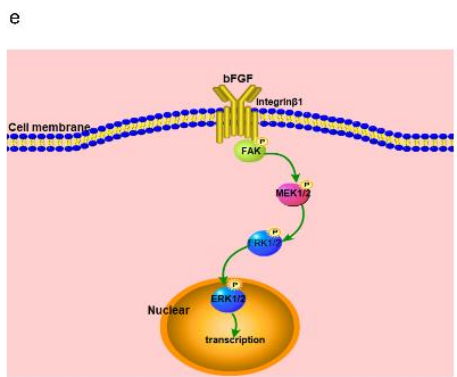

Fig 4: MEK1/2 suppression decreased Collagen expression. Control group: L929 cells. U0126 group: L929 cells dealt with U0126. DMSO group: U0126 negative control group. bFGF+ Control group: L929 cells cultivated by 2ng/ml bFGF. bFGF+ U0126 group: L929 cells dealt with U0126 and cultivated by $2 \mathrm{ng} / \mathrm{ml}$ bFGF. bFGF+ DMSO group: L929 cells dealt with DMSO and cultivated by $2 \mathrm{ng} / \mathrm{ml}$ bFGF.a, b: The fluorescence expression of Collagen III and Collagen I after MEK1/2 suppression. $c$, d: The protein expression of Collagen III and Collagen I after MEK1/2 suppression. * represents $\mathrm{p}<0.05,{ }^{* *}$ represents $\mathrm{p}<0.01, * * *$ represents $\mathrm{p}<0.001$.e: The schematic diagram of the cell signaling pathway in this paper. 


\section{Discussion}

The relaxation of supporting tissues of the pelvic floor is closely related to the incidence of POP[24]. The supporting tissue of the pelvic floor mainly includes fibrous components (collagen and elastin) and viscoelastic matrix (proteoglycan and glycoprotein), both of which are the main components of the extracellular matrix (ECM)[22]. Recently, ECM refactoring has been increasingly recognized as an important mechanism for the occurrence and development of POP $[25 ; 26]$. As an important component of the ECM, collagen is closely related to the occurrence and development of POP. Our experiments showed that the expression of collagen III and collagen I was significantly reduced in the ligaments of patients with POP, which is consistent with the research results of Gong, R[27]. Stimulating collagen regeneration is an effective way to improve pelvic floor function, and our previous studies have shown that electrical stimulation can effectively improve pelvic floor function by promoting collagen regeneration.

bFGF is a growth factor that can be secreted by pelvic floor fibroblasts, is combined with biological scaffolds and is widely used in tissue engineering. Many scholars have explored its effect on pelvic floor repair[28] and believe that it can be applied to rescue pelvic floor function. Previous research has shown that fibroblast apoptosis increased, cell function decreased, cell secretion decreased, and the extracellular matrix changed with the development of POP[18]. In our experiment, we measured the content of bFGF in the sacral ligament, and the results showed that there was a decrease in bFGF in patients with POP, which may be related to the decreased number and activity of fibroblasts in the vaginal wall of these patients and the decreased secretion of bFGF because the sacral ligament is an important supporting structure and its main cellular component is fibroblasts. In addition, our results showed that collagen increased significantly after bFGF stimulation of L929 cells. Therefore, we concluded that the reduction in bFGF is probably an important factor in the low collagen expression.

It is very difficult to extract primary fibroblasts from humans, and short cycles of primary cells are not sufficient to complete virus transfection. L929 cells were chosen to explore the specific mechanism of the influence of bFGF on collagen. L929 cells have a stable system, grow rapidly and are commonly used in the study of POP[7]. We first conducted cell proliferation and apoptosis experiments and found that a low dose of bFGF inhibited apoptosis and promoted proliferation, while excessive bFGF showed the opposite effect; a low dose of bFGF may promote the cell growth signal transduction pathway, while a high dose of bFGF has a toxic effect. However, we only observed the effects through flow cytometric analysis of apoptosis and did not detect changes in apoptotic proteins. Because the bFGF content in normal human tissues is extremely low, we hypothesized that the content of bFGF in pelvic floor tissues cannot reach a level that induces damage.

Used an optimal concentration of bFGF $(2 \mathrm{ng} / \mathrm{ml})$ to investigate the signal transduction mechanism of bFGF promoting collagen production at the cellular level. As expected, the protein expression of p-MEK $1 / 2$ and p-ERK1/2 increased significantly after bFGF stimulation. We also observed a significant increase in integrin $\beta 1$, which is very interesting. Therefore, we induced integrin $\beta 1$ 
overexpression and silencing with lentivirus. We found that the overexpression or silencing of integrin $\beta 1$ can enhance or weaken the MEK1/2/ERK1/2 signaling pathway, which eventually leads to increased or decreased collagen expression. This is a relatively important finding that has not been proven. Therefore, we believe that bFGF can promote collagen expression through the integrin $\beta 1 / \mathrm{MEK} 1 / 2 / \mathrm{ERK} 1 / 2$ pathway.

Integrin $\beta 1$ is an important cell surface receptor that belongs to the transmembrane receptor family, and its $\beta$ subunit can participate in intracellular signal transduction and regulate the biological functions of cells $[29 ; 30 ; 31]$. Studies have shown that integrins can be directly activated by growth factors, and cells can be activated and adhered to by the aggregation of integrins[32; 33]. The MEK1/2/ERK1/2 signal transduction pathway is closely related to collagen expression[21; 34]. In our study, we observed that the upregulation of integrin $\beta 1$ increased the mRNA levels of MEK1/2 and ERK1/2 and enhanced their phosphorylation. Therefore, we identified integrin $\beta 1$ as an upstream protein of bFGF acting on MEK1/2, which plays an important role in regulating collagen production. The main process may involve integrin $\beta 1$ activation after bFGF acts on its extracellular region, and then, the cytoplasmic terminal of the integrin $\beta 1$ subunit binds to the $\mathrm{N} 2$ terminal region of FAK, an important member of the signaling pathway mediated by integrin, which causes the conformational change and activates the kinase domain of FAK. Fully activated FAK enters the Ras pathway through acidified paxillin and the tyrosine residue of Cas, Then, Ras can further activate Raf to phosphorylate MEK1/2 and phosphorylated MEK1/2 further activates ERK1/2, which results in a cascade of reactions leading to tertiary activation of the MAPK pathway[35]. Phosphorylated ERK1/2 is transferred into the nucleus to regulate transcription and finally control collagen production.

In conclusion, our experiments first revealed that bFGF promotes the expression of collagen in L929 cells by stimulating the integrin $\beta 1 /$ MEK1/2/ERK1/2 signal transduction pathway. However, signal transduction is very complex, and this pathway may be just one of several pathways. It is a pity that there is no animal model of POP disease to do some animal studies Currently. In summary, this discovery may contribute to the application of bFGF in pelvic floor studies.

\section{Acknowledgements}

This work was financially supported by the National Natural Science Foundation of China (no. 81971364, 81771562), Second level fund of the second medical leading talents project of Hubei province (no. [2019]47), National key research and development program (2018YFC2002204), Special project for Chinese women's pelvic floor dysfunction prevention and treatment (no. 201817092) and the Natural Science Foundation of Hubei Province (no.2019CFB149).

\section{Author contributions}

Yang Lian and Hong Shasha contributed equally. Yang Lian and Hong Shasha: Design experiments, Experiment, Analyze data, and write a paper. Li Yang: Data Curation and analysis dates.Wang Tingting: Collating of data.Li Lu: Collating of data Hong Li: Financial and technical support.

\section{Competing of interest}


The authors declare that they have no conflict of interest.

\section{Ethical approval}

The study protocol and patient informed consent have been approved by the local ethics committee and are following the Declaration of Ethics Committee of People's Hospital of Wuhan University.

\section{Informed consent}

Informed consent was obtained from all individual participants included in the study.

\section{Statement}

All methods in this work were carried out in accordance with relevant guidelines and regulations.

\section{References:}

[1] R.C. Bump, A. Mattiasson, K. Bo, et al., The standardization of terminology of female pelvic organ prolapse and pelvic floor dysfunction, AM J OBSTET GYNECOL 175 (1996)10-7.

[2] A.S. Lin, S. Carrier, D.M. Morgan, et al., Effect of simulated birth trauma on the urinary continence mechanism in the rat, UROLOGY 52 (1998)143-51.

[3] T. Tyagi, M. Alarab, Y. Leong, et al., Local oestrogen therapy modulates extracellular matrix and immune response in the vaginal tissue of post-menopausal women with severe pelvic organ prolapse, $\mathrm{J}$ CELL MOL MED 23 (2019)2907-2919.

[4] H. Wang, Y. Kira, A. Hamuro, et al., Differential gene expression of extracellular-matrix-related proteins in the vaginal apical compartment of women with pelvic organ prolapse, INT UROGYNECOL J 30 (2019)439-446.

[5] Y. Li, B.S. Li, C. Liu, et al., Effect of integrin beta1 in the treatment of stress urinary incontinence by electrical stimulation, MOL MED REP 19 (2019)4727-4734.

[6] S. Elena, Z. Dragana, S. Ramina, et al., Electromyographic Evaluation of the Pelvic Muscles Activity After High-Intensity Focused Electromagnetic Procedure and Electrical Stimulation in Women With Pelvic Floor Dysfunction, Sex Med (2020).

[7] Y. Li, C. Liu, B. Li, et al., Electrical stimulation activates calpain 2 and subsequently upregulates collagens via the integrin beta1/TGF-beta1 signaling pathway, CELL SIGNAL 59 (2019)141-151.

[8] D. Gospodarowicz, K.L. Jones, G. Sato, Purification of a growth factor for ovarian cells from bovine pituitary glands, Proc Natl Acad Sci U S A 71 (1974)2295-9.

[9] A.O. Wilkie, S.J. Patey, S.H. Kan, et al., FGFs, their receptors, and human limb malformations: clinical and molecular correlations, Am J Med Genet 112 (2002)266-78.

[10] H. Zhang, K. Wang, T. Gao, et al., Controlled release of bFGF loaded into electrospun core-shell fibrous membranes for use in guided tissue regeneration, BIOMED MATER (2020).

[11] H. Zhang, K. Wang, T. Gao, et al., Controlled release of bFGF loaded into electrospun core-shell fibrous membranes for use in guided tissue regeneration, BIOMED MATER (2020).

[12] X. Xuan, Y. Zhou, A. Chen, et al., Silver crosslinked injectable bFGF-eluting supramolecular hydrogels speed up infected wound healing, J MATER CHEM B 8 (2020)1359-1370. 
[13] S.G. Hansen, M.B. Taskin, M. Chen, et al., Electrospun nanofiber mesh with fibroblast growth factor and stem cells for pelvic floor repair, J Biomed Mater Res B Appl Biomater 108 (2020)48-55.

[14] D. Zhang, Z. Lin, R. Cheng, et al., Reinforcement of transvaginal repair using polypropylene mesh functionalized with basic fibroblast growth factor, Colloids Surf B Biointerfaces 142 (2016)10-19.

[15] Y.Y. Jia, J.Y. Zhou, Y. Chang, et al., Effect of Optimized Concentrations of Basic Fibroblast Growth Factor and Epidermal Growth Factor on Proliferation of Fibroblasts and Expression of Collagen: Related to Pelvic Floor Tissue Regeneration, Chin Med J (Engl) 131 (2018)2089-2096.

[16] H. Madry, D. Kohn, M. Cucchiarini, Direct FGF-2 gene transfer via recombinant adeno-associated virus vectors stimulates cell proliferation, collagen production, and the repair of experimental lesions in the human ACL, Am J Sports Med 41 (2013)194-202.

[17] Y. Wang, C. Fu, Z. Wu, et al., A chitin film containing basic fibroblast growth factor with a chitin-binding domain as wound dressings, Carbohydr Polym 174 (2017)723-730.

[18] Q. Li, B. Li, C. Liu, et al., Protective role of Nrf2 against mechanical-stretch-induced apoptosis in mouse fibroblasts: a potential therapeutic target of mechanical-trauma-induced stress urinary incontinence, INT UROGYNECOL J 29 (2018)1469-1477.

[19] H. Qiang, Y. Lin, X. Zhang, et al., Differential expression genes analyzed by cDNA array in the regulation of rat hepatic fibrogenesis, LIVER INT 26 (2006)1126-37.

[20] R. Jaster, G. Sparmann, J. Emmrich, et al., Extracellular signal regulated kinases are key mediators of mitogenic signals in rat pancreatic stellate cells, GUT 51 (2002)579-84.

[21] Y. Nagai, K. Miyata, G.P. Sun, et al., Aldosterone stimulates collagen gene expression and synthesis via activation of ERK1/2 in rat renal fibroblasts, HYPERTENSION 46 (2005)1039-45.

[22] H. Peng, O.A. Carretero, E.L. Peterson, et al., N-Acetyl-seryl-aspartyl-lysyl-proline inhibits ET-1-induced collagen production by preserving Src homology 2-containing protein tyrosine phosphatase-2 activity in cardiac fibroblasts, Pflugers Arch 464 (2012)415-23.

[23] Y. Li, C. Liu, B. Li, et al., Electrical stimulation activates calpain 2 and subsequently upregulates collagens via the integrin beta1/TGF-beta1 signaling pathway, CELL SIGNAL 59 (2019)141-151.

[24] S.G. Hansen, M.B. Taskin, M. Chen, et al., Electrospun nanofiber mesh with fibroblast growth factor and stem cells for pelvic floor repair, J Biomed Mater Res B Appl Biomater (2019).

[25] J. Tang, B. Li, C. Liu, et al., Mechanism of Mechanical Trauma-Induced Extracellular Matrix Remodeling of Fibroblasts in Association with Nrf2/ARE Signaling Suppression Mediating TGF-beta1/Smad3 Signaling Inhibition, OXID MED CELL LONGEV 2017 (2017)8524353.

[26] G. Lin, A.W. Shindel, L. Banie, et al., Molecular mechanisms related to parturition-induced stress urinary incontinence, EUR UROL 55 (2009)1213-22.

[27] R. Gong, Z. Xia, Collagen changes in pelvic support tissues in women with pelvic organ prolapse, Eur J Obstet Gynecol Reprod Biol 234 (2019)185-189.

[28] S.G. Hansen, M.B. Taskin, M. Chen, et al., Electrospun nanofiber mesh with fibroblast growth factor and stem cells for pelvic floor repair, J Biomed Mater Res B Appl Biomater 108 (2020)48-55.

[29] I.D. Campbell, M.J. Humphries, Integrin structure, activation, and interactions, Cold Spring Harb Perspect Biol 3 (2011).

[30] G. Gruber, J. Hess, C. Stiefel, et al., Correlation between the tumoral expression of beta3-integrin and outcome in cervical cancer patients who had undergone radiotherapy, Br J Cancer 92 (2005)41-6.

[31] G. Gruber, J. Hess, C. Stiefel, et al., Correlation between the tumoral expression of beta3-integrin and outcome in cervical cancer patients who had undergone radiotherapy, Br J Cancer 92 (2005)41-6.

[32] B.P. Eliceiri, Integrin and growth factor receptor crosstalk, CIRC RES 89 (2001)1104-10. 
[33] L. Feng, Y. Li, W. Zeng, et al., Enhancing effects of basic fibroblast growth factor and fibronectin on osteoblast adhesion to bone scaffolds for bone tissue engineering through extracellular matrix-integrin pathway, EXP THER MED 14 (2017)6087-6092.

[34] X. Wu, M. Li, S.Q. Chen, et al., Pin1 facilitates isoproterenolinduced cardiac fibrosis and collagen deposition by promoting oxidative stress and activating the MEK1/2ERK1/2 signal transduction pathway in rats, INT J MOL MED 41 (2018)1573-1583.

[35] S.Y. Cheng, G. Sun, D.D. Schlaepfer, et al., Grb2 promotes integrin-induced focal adhesion kinase (FAK) autophosphorylation and directs the phosphorylation of protein tyrosine phosphatase alpha by the Src-FAK kinase complex, MOL CELL BIOL 34 (2014)348-61. 
Figures
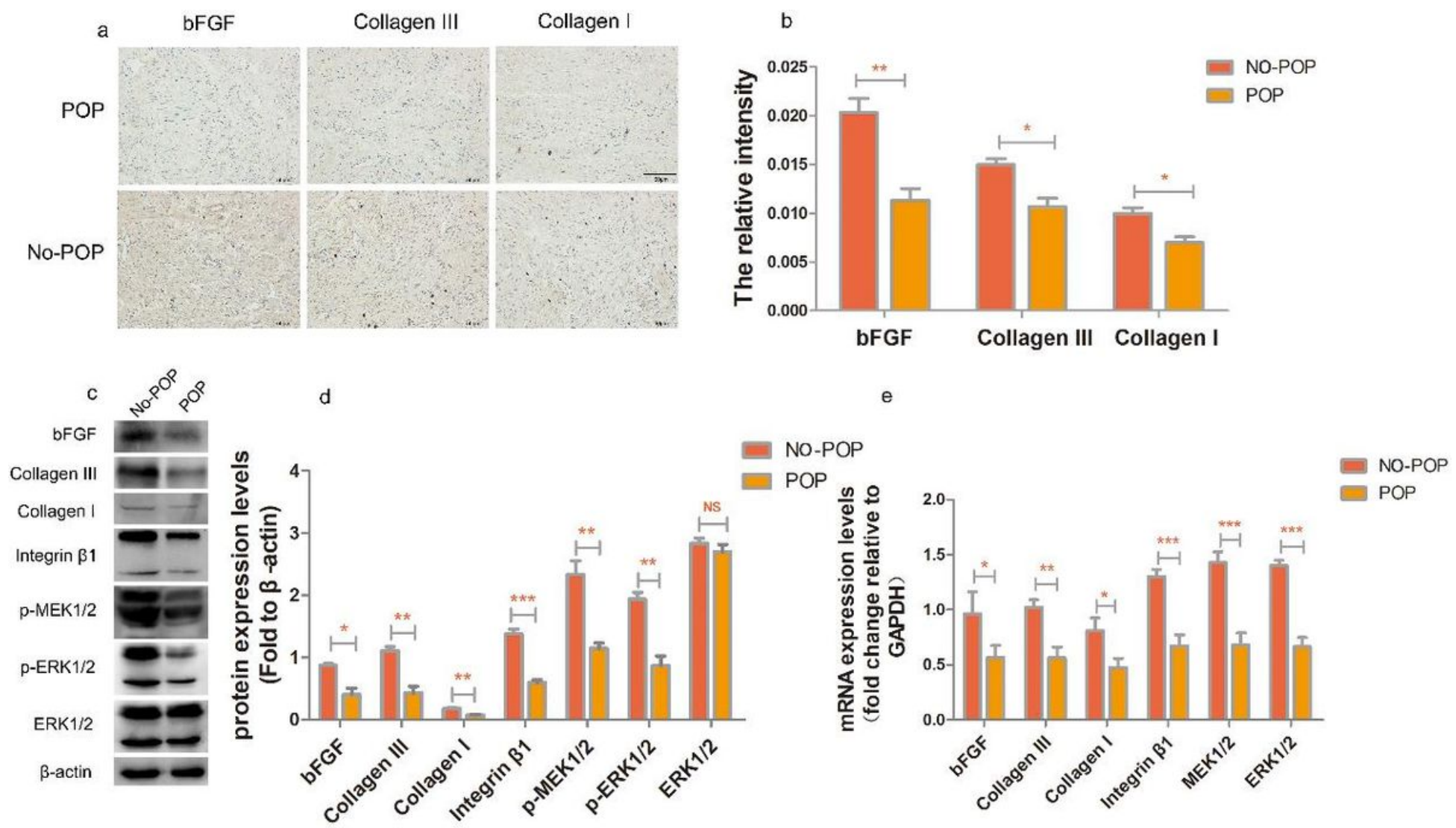

Figure 1

The expression of bFGF, Collagen III, Collagen I, Integrin $\beta 1$, p-MEK1/2, and p-ERK1/2 in POP patients was lower than NO-POP patients( POP group: POP patients; NO-POP: NO-POP patients. a:

Immunohistochemical staining of the sacral ligament, amplification factor: $\times 200$. b: Fluorescence expression analysis of bFGF, Collagen III, and Collagen I. c: Protein expression of bFGF, CollagenIII, Collagen I, Integrin $\beta 1$, p-MEK1/2 and p-ERK1/2 in vaginal wall tissues. d: Statistical analysis of western blots. e: mRNA expression of bFGF, CollagenIII, Collagen I, Integrin $\beta 1$, p-MEK1/2, p-ERK1/2, and ERK1/2 in vaginal wall tissues. * represents $p<0.05$, ** represents $p<0.01$, *** represents $p<0.001$, NS represents no significance, Each experiment was repeated three times. 

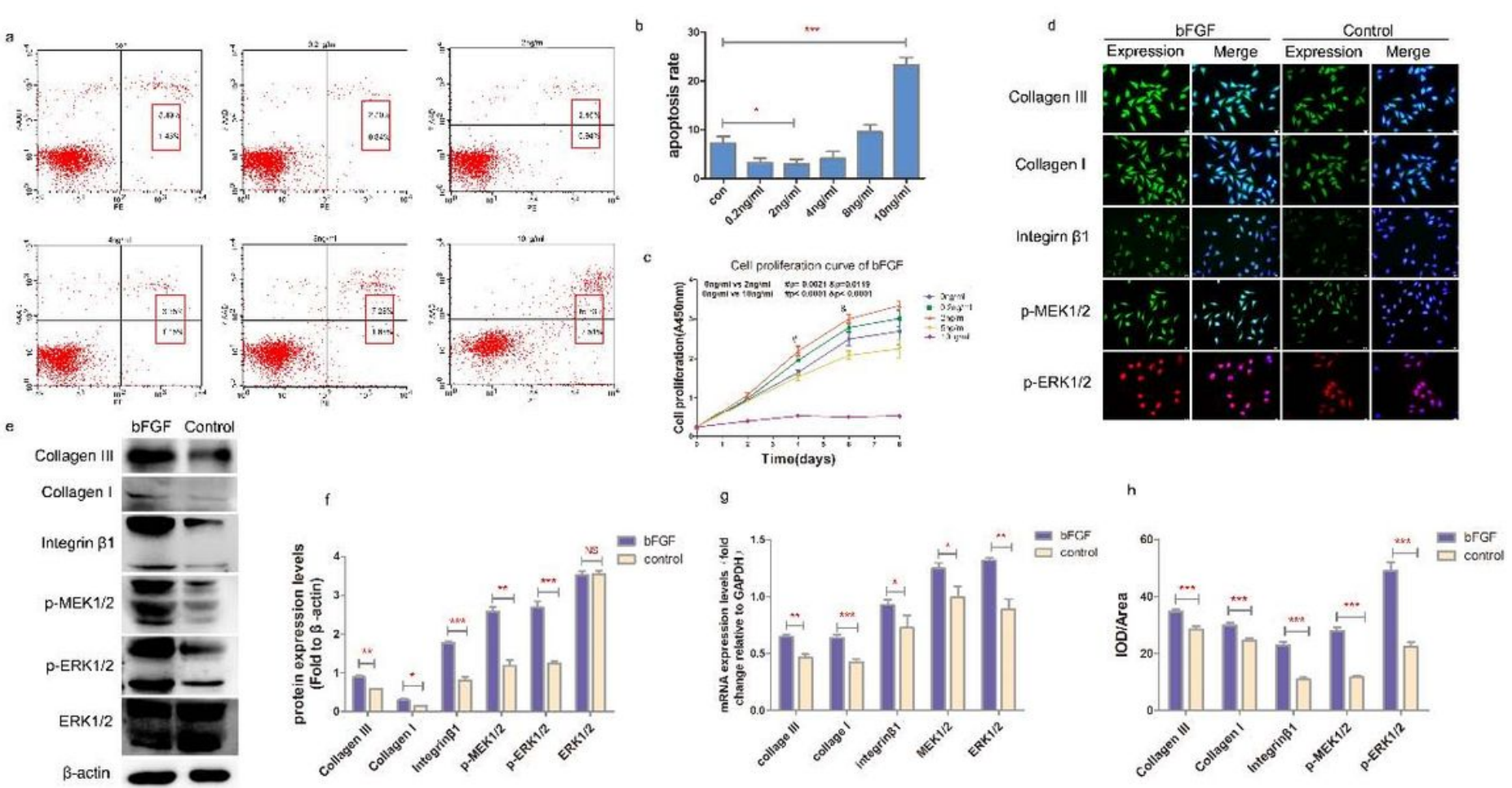

\section{Figure 2}

bFGF stimulation promoted the expression of Collagen and Integrin $\beta 1$ and enhance the phosphorylation of MEK1/2 and ERK1/2 a: Flow diagram of apoptosis; b: Statistical analysis apoptosis rate of $0 \mathrm{ng} / \mathrm{ml}$, $0.2 \mathrm{ng} / \mathrm{ml}, 2 \mathrm{ng} / \mathrm{ml}, 4 \mathrm{ng} / \mathrm{ml}, 8 \mathrm{ng} / \mathrm{ml}$ and $10 \mathrm{ng} / \mathrm{ml}$, c: cell proliferation curve.d, h: Immunofluorescence staining and expression of cells stimulated by bFGF, amplification factor: $\times 400 ; e$, ,: Protein expression of CollagenIII, collagen I, Integrin 1 , p-MEK1/2, p-ERK1/2, and ERK1/2 after bFGF stimulation; g: mRNA expression of CollagenIII, collagen I, integrin $\beta 1$, p-MEK1/2, p-ERK1/2 and ERK1/2 after bFGF stimulation. * represents $p<0.05$, ** represents $p<0.01$, *** represents $p<0.001$, NS represents no significance. 


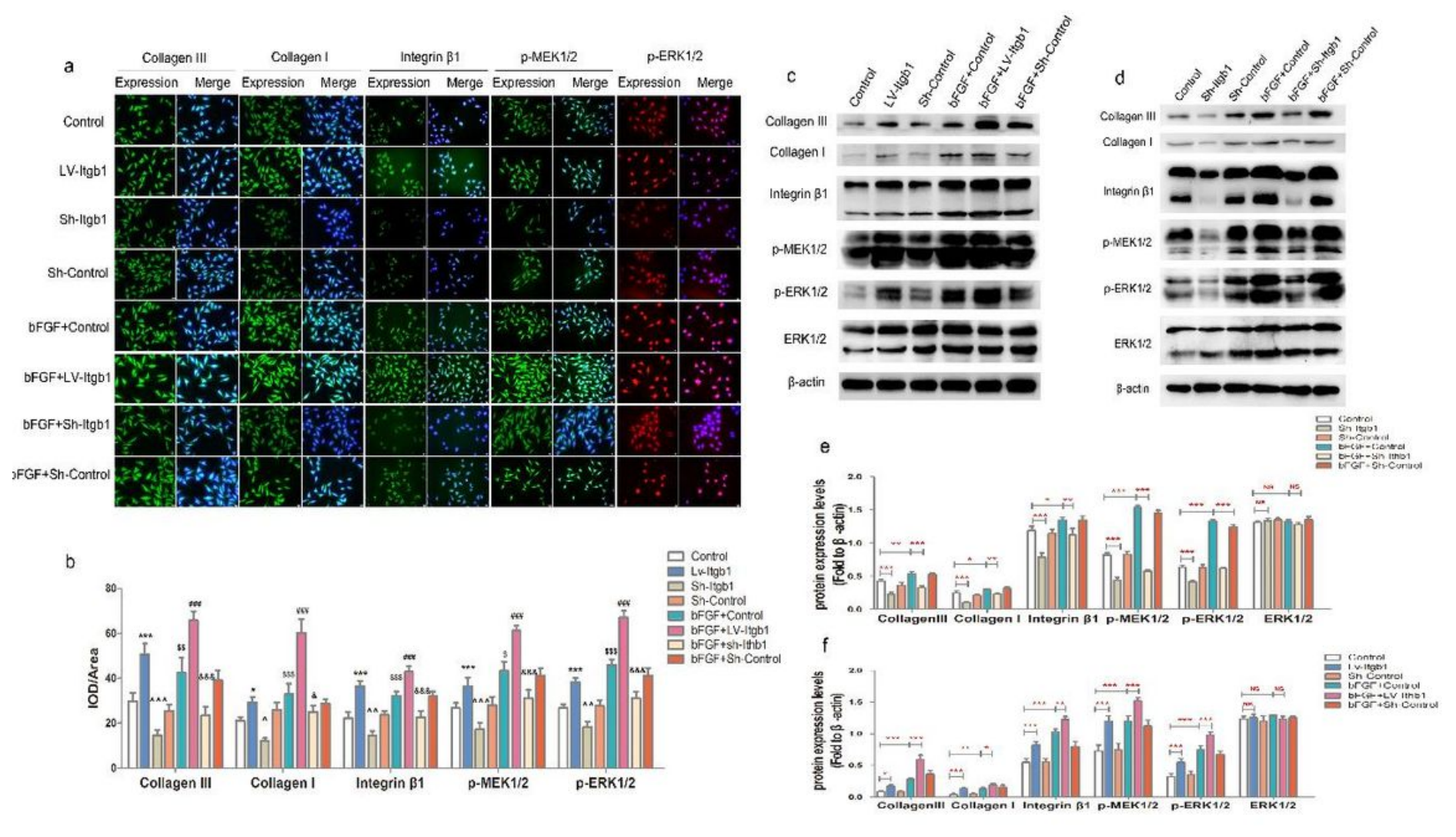

Figure 3

Fluorescence expression of CollagenIII, Collagen I, Integrin $\beta 1$, p-MEK1/2, P-ERK1/2 in cells after overexpression and silencing of integrin $\beta 1$. Control group: L929 cells cultivated by Conventional medium. LV-Itgb1: Integrins overexpress lentivirus transfected L929 cells. Sh-Itgb1: Integrins silence lentivirus transfected L929 cells. Sh-Control group: Lentivirus negative control group.bFGF+Control group: L929 cells cultivated by $2 \mathrm{ng} / \mathrm{ml}$ bFGF. bFGF+ LV-ltgb1 group: Integrins overexpress lentivirus transfected L929 cells and cultivated by 2ng/ml bFGF. bFGF+Sh-ltgb1 group: Integrins silence lentivirus transfected L929 cells and cultivated by $2 \mathrm{ng} / \mathrm{ml}$ bFGF. bFGF+Sh-Control group: Lentivirus negative control group cultivated by $2 \mathrm{ng} / \mathrm{ml}$ bFGF. a: Fluorescence staining diagram, magnification factor: $\times 400$. b: Fluorescence expression analysis. *represents for Control VS Lv-ltgb1, ^ represents for control VS Sh-Itgb1, \$ represents for Control VS bFGF+control, \# represents bFGF+Control VS bFGF+ Lv-Itgb1, \& represents bFGF+Control VS bFGF+ Sh-Itgb1. c, f : Protein expression after integrin $\beta 1$ overexpression. d,e: Protein expression after integrin $\beta 1$ silence. A marker represents $p<0.05$, Two marks indicate $p<0.01$, Three markers indicate $p$ $<0.001$, NS represents no significance. 
a
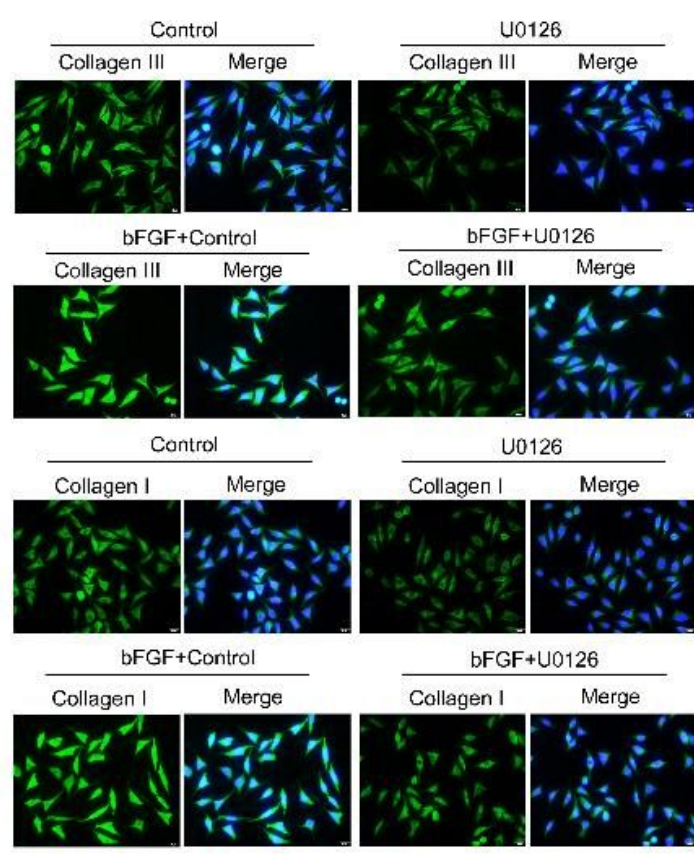

FGF+U0126
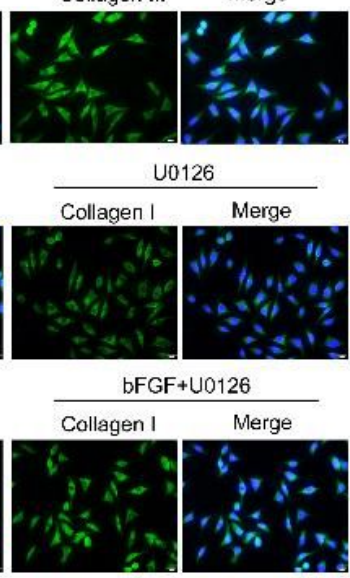

d

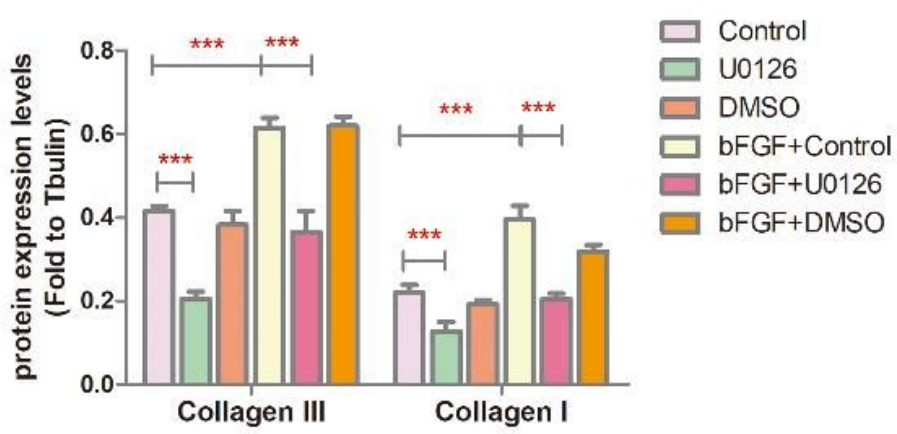

b
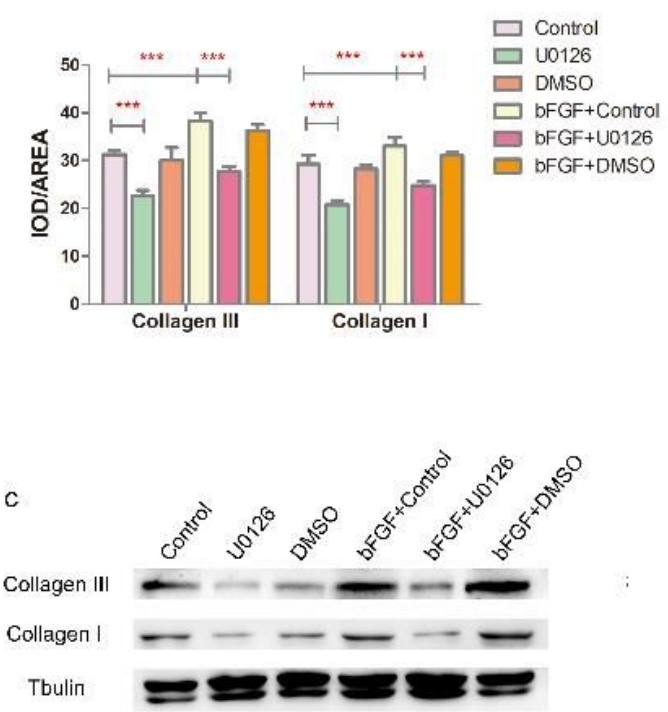

e

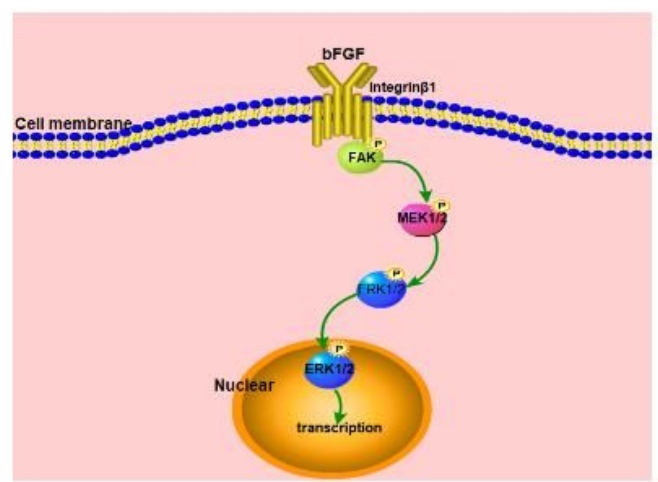

\section{Figure 4}

MEK1/2 suppression decreased Collagen expression. Control group: L929 cells. U0126 group: L929 cells dealt with U0126. DMSO group: U0126 negative control group. bFGF+ Control group: L929 cells cultivated by $2 \mathrm{ng} / \mathrm{ml}$ bFGF. bFGF+ U0126 group: L929 cells dealt with U0126 and cultivated by $2 \mathrm{ng} / \mathrm{ml}$ bFGF. bFGF+ DMSO group: L929 cells dealt with DMSO and cultivated by $2 \mathrm{ng} / \mathrm{ml}$ bFGF.a, b: The fluorescence expression of Collagen III and Collagen I after MEK1/2 suppression. c, d: The protein expression of Collagen III and Collagen I after MEK1/2 suppression. * represents $p<0.05$, ** represents $p<0.01$, *** represents $p<0.001$.e: The schematic diagram of the cell signaling pathway in this paper.

\section{Supplementary Files}

This is a list of supplementary files associated with this preprint. Click to download.

- Supplementarymaterial.pdf 